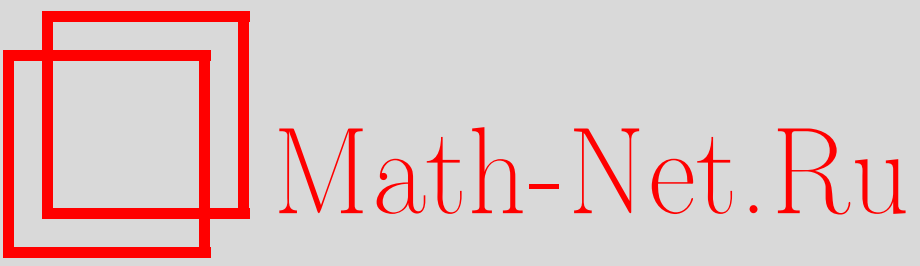

В. И. Корнеев, Н. Е. Кулагин, А. Ф. Попков, Нелинейные локализованные волны в среде с нелокальным взаимодействием, ТМФ, 1998, том 114, номер 3, 366-379

DOI: https://doi.org/10.4213/tmf846

Использование Общероссийского математического портала Math-Net.Ru подразумевает, что вы прочитали и согласны с пользовательским соглашением

http://www . mathnet.ru/rus/agreement

Параметры загрузки:

IP : 54.198 .64 .247

26 апреля 2023 г., 10:58:01 
ТЕОРЕТИЧЕСКАЯ

И МАТЕМАТИЧЕСКАЯ

ФИЗИКА

Том 114, № 3

март, 1998

(C) 1998 г. $\quad$ В.И. Корнеев ${ }^{*}$ Н.Е. Кулагин ${ }^{\dagger}$, А. Ф. Попков ${ }^{\dagger}$

\section{НЕЛИНЕЙНЫЕ ЛОКАЛИЗОВАННЫЕ ВОЛНЫ В СРЕДЕ С НЕЛОКАЛЬНЫМ ВЗАИМОДЕЙСТВИЕМ}

Найдены солитонные решения нелинейных интегродифференциальных уравнений с ядром типа $\lambda /\left(\tau-\tau^{\prime}\right)$, используемых для описания туннелирования частиц, магнитных и сверхпроводящих вихрей в среде с нелокальным взаимодействием. Методом фурье-преобразования получена асимптотика четных и нечетных локализованных решений. Найдены аналитические решения при частных значениях параметров. Методом численного расчета получена полная картина поведения солитонных решений в произвольной области изменения параметра взаимодействия $\lambda$.

\section{1. ВВЕДЕНИЕ}

Локализованные во времени либо в пространстве волны в нелинейных средах с нелокальным взаимодействием привлекают в последнее время внимание исследователей в различных областях физики. К ним относятся, например, джозефсоновские флуксоны в распределенном сверхпроводяшем контакте с большим критическим током [1-5], инстантоны туннелирования в точечном сверхпроводящем контакте [6, 7], инстантоны в магнитных мезочастицах [8-10] и солитоны в распределенных спиновых системах [11-13]. Общим свойством таких нелинейных систем является то, что в конечном итоге их описание можно свести к нелинейному уравнению для одной динамической переменной с интегральным ядром, которое эквивалентно уравнению движения инерциальной частицы в среде с нелокальным взаимодействием, а именно

$$
M q_{\tau \tau}=\frac{d}{d q} V(q)+\frac{1}{2} \int_{-\infty}^{\infty} G\left(\tau-\tau^{\prime}\right) q_{\tau^{\prime}} d \tau^{\prime}
$$

где $M$ - масса частицы, $V(q)$ - нелинейная потенциальная функция, $G\left(\tau-\tau^{\prime}\right)$ - ядро, учитывающее динамический отклик среды. Роль динамической переменной $q(\tau)$, массы и других параметров в уравнении (1) выполняют различные физические параметры и их комбинации в зависимости от типа среды. В случае распределенного джозефсоновского контакта с большим отношением лондоновской $\lambda_{\mathrm{L}}$ и джозефсоновской $\lambda_{\mathrm{J}}$ глубин экранировки электродинамика описывается нелокальным уравнением “синус-Гордон” $[1,2]$. В

\footnotetext{
* Московский институт электронной техники, Зеленоград, Москва, Россия

${ }^{\dagger}$ Научно-исследовательский институт физических проблем, Зеленоград, Москва, Россия
} 
автомодельном волновом приближении, когда структура локализованной волны (флуксона) зависит от координаты $x$ и времени $t$ только в комбинации $t=x-v t$, где $v-$ скорость флуксона, роль массы выполняет величина $M=-C(\hbar v / 2 e)^{2}$, где $C$ - погонная емкость, $\hbar$ - постоянная Планка, $e$ - заряд электрона. Динамической переменной при этом является скачок фазы сверхпроводящих электронов на переходе. Потенциальная энергия представляет собой энергию сверхпроводящего контакта

$$
V(q)=V_{0}(1-\cos q),
$$

где $V_{0}=j_{c} \hbar / 2 e$, а $j_{c}$ - линейная плотность критического тока. Ядро $G\left(\tau-\tau^{\prime}\right)$ определяется эффектом экранировки магнитного поля флуксона в берегах перехода и в пределе $\lambda_{\mathrm{L}} / \lambda_{\mathrm{J}} \rightarrow \infty$ имеет простой вид:

$$
G=\frac{V_{0} \lambda_{\mathrm{J}}^{2}}{\pi \lambda_{\mathrm{L}}\left(\tau-\tau^{\prime}\right)} .
$$

Уравнение (1) в данном случае после перенормировок принимает следующий вид:

$$
-q_{x x}=\sin q+\lambda \int_{-\infty}^{\infty} \frac{q_{x^{\prime}} d}{x-x^{\prime}} d x^{\prime}
$$

где $x=\tau\left(V_{0} /|M|\right)^{1 / 2}, \lambda=\lambda_{\mathrm{J}}^{2} / e \pi \lambda_{\mathrm{L}}\left(V_{0} /|M|\right)^{1 / 2}$.

Другим важным примером системы с нелокальным взаимодействием являются инстантоны в среде с диссипативным окружением, которые описываются уравнением Калдейры-Леггетта [6]

$$
M q_{\tau \tau}=\frac{d}{d q} V(q)+\frac{1}{2} \int_{-\infty}^{\infty} \alpha\left(\tau-\tau^{\prime}\right)\left(q(\tau)-q\left(\tau^{\prime}\right)\right) d \tau^{\prime}
$$

где интегральное ядро $\alpha\left(\tau-\tau^{\prime}\right)$ определяется характером динамического отклика среды. Если $q(\tau)$ достаточно быстро убывает на бесконечности, то после интегрирования по частям уравнение (3) сводится к уравнению (1). Уравнение (3) использовалось для описания макроскопического квантового туннелирования электронов в точечном сверхпроводяшем контакте. В этом случае роль массы играет нормированная емкость контакта $M=C(\hbar / 2 e)^{2}$, а $\tau$ - мнимое время. При смещении контакта внешним током $I$, близким к критическому $I_{c}$, потенциальная функция имеет следующую зависимость:

$$
V(q)=\frac{27}{4} V_{0}\left[\left(\frac{q}{q_{0}}\right)^{2}-\left(\frac{q}{q_{0}}\right)^{3}\right],
$$

где $V_{0}=2 I_{c} \hbar / 32 e, q_{0}=\left(1-\left(I / I_{c}\right)^{2}\right)^{1 / 3}$. Ядро оператора $\alpha\left(\tau-\tau^{\prime}\right)$ зависит от частотной характеристики диссипативного окружения $J(\omega)$, т.е.

$$
\alpha\left(\tau-\tau^{\prime}\right)=\frac{1}{2 \pi} \int_{0}^{\infty} J(\omega) e^{-\omega\left|\tau-\tau^{\prime}\right|} d \omega .
$$

В случае “омического" окружения, когда $J(\omega)=\eta \omega$, ядро имеет вид

$$
\alpha\left(\tau-\tau^{\prime}\right)=\frac{\eta}{2 \pi\left(\tau-\tau^{\prime}\right)}
$$


При этом в сверхпроводящем контакте с шунтирующей проводимостью $\sigma$ диссипативный параметр равен $\eta=\sigma(\hbar / 2 e)^{2}$. В нормированных переменных уравнение $(3)$ в этом случае имеет вид

$$
u_{x x}=u-\frac{3}{2} u^{2}+\lambda \int_{-\infty}^{\infty} \frac{u_{x^{\prime}}}{x-x^{\prime}} d x^{\prime}
$$

где $u=q / q_{0}, x=\tau\left(27 V_{0} / 2 M q_{0}^{2}\right)^{1 / 2}, \lambda=\left(\eta q_{0} / 4 \pi\right)\left(2 / 27 V_{0} M\right)^{1 / 2}$.

В последнее время аналогичные уравнения используются при анализе туннелирования намагниченности в малых ферромагнитных частицах [8-10]. В случае двухосной анизотропии магнитной частицы, помещенной в магнитное поле $H$, параллельное легкой оси (ЛО) $(H \| Л О)$ и близкое по величине к критическому значению $H_{c}=2 K_{1} / M_{0}$, где $M_{0}$ - намагниченность и $K_{1}$ - энергия магнитокристаллической одноосной анизотропии, роль массы в уравнении (1) играет величина $M=M_{0}^{2} / 2 \gamma^{2} K_{2}$, где $\gamma$ - магнитомеханическое отношение, $K_{2}$ - энергия базисной анизотропии. При этом динамической переменной является полярньй угол вектора намагниченности $q=\theta$, а потенциальная энергия определяется формулой

$$
V(q)=4 V_{0}\left[\left(\frac{q}{q_{0}}\right)^{2}-\left(\frac{q}{q_{0}}\right)^{4}\right],
$$

где $V_{0}=K_{1}\left(1-H / H_{c}\right)^{2}, q_{0}=2\left(1-H / H_{c}\right)^{1 / 2}$. В случае $H \perp$ ЛО имеем соответственно

$$
V(q)=V_{0}\left[1-\left(\frac{q}{q_{0}}\right)^{2}\right]^{2}
$$

где $M=M_{0}^{2} / 2 \gamma^{2} K_{1}, q_{0}=\left(2\left(1-H / H_{c}\right)\right)^{1 / 2}$. Что касается ядра интегрального уравнения (1) для подобного случая, то в [9] рассмотрено фононное диссипативное окружение, для которого $J(\omega)=A\left(\omega / \omega_{c}\right)^{3} \exp \left(-\omega / \omega_{c}\right)$, где $A$ определяется упругими и магнитоупругими модулями среды, а частота отсечки равна $\omega_{c}=s / r$, где $s-$ скорость звука, $r$ - радиус частицы. Следует заметить, что диссипация в магнитной среде не всегда определяется только спин-фононным процессом релаксации, и поэтому естественно рассмотреть также случай “омического” окружения, когда частотный отклик магнитной среды носит линейный характер $J(\omega)=\eta \omega$. Подобный характер малых колебаний намагниченности описывается уравнениями магнитодинамики в форме Гильберта [11]. При этом коэффициент релаксации $\eta$ легко связать с параметром затухания Гильберта $\alpha$ в уравнениях динамики намагниченности при малой амплитуде угловой переменной. Действительно, диссипативная функция для ферромагнетика имеет вид [11] $R=\left(\alpha M_{0} / 2 \gamma\right)\left(\dot{\theta}^{2}+\dot{\varphi}^{2} \sin ^{2} \theta\right)$, где $\theta, \varphi$ - полярный и азимутальный углы, характеризующие направление вектора намагниченности. Поэтому в ситуации, когда $\theta \ll 1, \varphi \ll 1$, приближенно имеем $R=\left(\alpha M_{0} / 2 \gamma\right) \dot{\theta}^{2}$, следовательно, $\eta=\alpha M_{0} / \gamma$. В последнем случае уравнение магнитных инстантонов в нормированных переменных согласно модели Калдейры-Леггетта $[6,10]$ принимает вид

$$
u_{x x}=\frac{d}{d u} V(u)+\lambda \int_{-\infty}^{\infty} \frac{u(x)-u\left(x^{\prime}\right)}{\left(x-x^{\prime}\right)^{2}} d x^{\prime},
$$


где $\lambda=\left(\eta q_{0} / 4 \pi\right)\left(V_{0} M\right)^{-1 / 2}$. При этом $V(u)=u^{2}-u^{4}$ в том случае, если макроскопическое квантовое туннелирование описывается в области магнитного гистерезиса вблизи критического значения поля, либо $V(u)=\left(1-u^{2}\right)^{2}$, если рассматривается явление макроскопической квантовой когерентности состояний с $u= \pm 1$ в перпендикулярном магнитном поле $H \perp$ ЛО.

Приведенные примеры показывают, что уравнения типа (1) описывают достаточно широкий круг физических явлений. Решение этих уравнений обычно строится приближенно. Точные решения получены только для некоторых частных случаев. В настояшей работе методом качественного и численного анализов находятся локализованные решения уравнений вида (5) для рассмотренных выше потенциалов $V(q)$. При этом мы не ограничились областью положительных значений параметра $\lambda$, а для обшности рассмотрели также область отрицательных значений, в которой возникают локализованные решения. Физическая ситуация, соответствующая подобному случаю, возникает, например, при рассмотрении магнитных вихрей в доменной границе магнитного материала с большой одноосной анизотропией [11-13]. Эта система описывается двумя дифференциальными уравнениями Слончевского [11], которые сводятся к интегродифференциальному уравнению типа (1) с экспоненциальным ядром. В обшем случае произвольного ядра уравнение (1) не сводится к конечной системе дифференциальных уравнений. То же касается, например, динамики магнитных солитонов и вихрей в среде с нелокальной магнитостатикой [13]. Поэтому анализ уравнений типа (1) в общем случае представляет несомненный интерес.

\section{2. УРАВНЕНИЯ С КВАДРАТИЧНОЙ НЕЛИНЕЙНОСТЬЮ}

Рассмотренные во введении уравнения можно записать в следующем виде:

$$
u_{x x}+g(u)=\lambda K[u], \quad K[u]=\frac{1}{\pi} \int_{-\infty}^{\infty} \frac{u(x)-u\left(x^{\prime}\right)}{\left(x-x^{\prime}\right)^{2}} d x^{\prime},
$$

где $g(u)$ - заданная нелинейная функция, $\lambda$ - безразмерный параметр, характеризующий степень воздействия нелокальности в системе, $x$ - безразмерная текущая координата, которая может выполнять роль как пространственной, так и временной переменной в зависимости от рассматриваемой физической ситуации.

Рассмотрим уравнение с квадратичной нелинейностью, в котором $g(u)=-u+u^{2}$, т.e.

$$
u_{x x}-u+u^{2}=\lambda K[u] .
$$

Для некоторых значений параметра $\lambda$ удается получить локализованные решения уравнения (7) в аналитическом виде. Если $\lambda=0$, то уравнение (7) не содержит нелокального члена и локализованное (солитонное) решение находится элементарно:

$$
u(x)=\frac{3}{2 \operatorname{ch}^{2}(x / 2)} .
$$

В случае $\lambda \neq 0$ прямой подстановкой можно показать, что имеется солитонное решение следующего вида:

$$
u(x)=\frac{A}{a^{2}+x^{2}}+\frac{B}{\left(a^{2}+x^{2}\right)^{2}},
$$


где коэффишиенты $A, B, a, \lambda$ находятся из условия обрашения (7) в тождество. В результате получаем

$$
\begin{aligned}
& A=12(\xi-1), \quad B=120 \frac{\xi^{2}}{\xi-1}, \quad a=\sqrt{B / 24}, \\
& \lambda=\frac{a A}{A+12}, \quad \xi=\sqrt[3]{(3+\sqrt{5}) / 2}+\sqrt[3]{(3-\sqrt{5}) / 2} .
\end{aligned}
$$

Приближенные значения этих параметров таковы: $A=13.3, \quad B=481, a=4.48$, $\lambda=2.35$.

При больших значениях параметра $\lambda$ решение уравнения (7) можно представить в виде разложения по малому параметру $1 / \lambda$ :

$$
u(x)=u_{0}(x / \lambda)+\frac{1}{\lambda} u_{1}(x / \lambda)+\cdots,
$$

где $u_{0}(\xi)$ удовлетворяет интегральному уравнению

$$
-u_{0}(\xi)+u_{0}^{2}(\xi)=\frac{1}{\pi} \int_{-\infty}^{\infty} \frac{u_{0}(\xi)-u_{0}\left(\xi^{\prime}\right)}{\left(\xi-\xi^{\prime}\right)^{2}} d \xi^{\prime}
$$

в котором переменная $\xi=x / \lambda$. Аналогично решению (9) можно показать, что

$$
u_{0}(\xi)=\frac{2}{1+\xi^{2}}
$$

Поэтому при больших значениях $\lambda$ получаем следуюшее решение уравнения (7):

$$
u(x)=\frac{2}{1+(x / \lambda)^{2}}+O(1 / \lambda) .
$$

Найденные частные решения были использованы для контроля численных решений.

Рассмотрим теперь случай произвольного $\lambda$. Будем искать численно локализованные четные решения $u(-x)=u(x)$ уравнения $(7)$ с асимптотикой $u(x) \sim c / x^{2}$, которая обосновывается ниже. Численный расчет проводится на отрезке $x \in[0, L]$ с граничными условиями $u_{x}(0)=0, u(L)=c / L^{2}$. Параметр $c$ вычисляется в процессе решения задачи. Разностная схема для уравнения (7) приводит к системе $N$ нелинейных алгебраических уравнений для $N$ неизвестных величин $u_{i}=u\left(x_{i}\right)$ в $N$ узловых точках $x_{i}=i L /(N-1), i=0,1, \ldots, N-1$. Часть интеграла $K[u]$, находящаяся вне интервала $[0, L]$, вычисляется аналитически с использованием асимптотического выражения $u(x)=c / x^{2}, x \geq L$. Особенность в интеграле (7) устраняется с точностью до $O\left(\Delta x^{2}\right)$, где $\Delta x=x_{i+1}-x_{i}$, т.е. с такой же точностью, с которой вычисляется вторая производная $u_{x x}$. Величина отрезка $L$ и число точек $N$ в разностной схеме определяются точностью расчета и для разных значений параметра $\lambda$ могут быть разными. Система полученных нелинейных разностных уравнений решается с использованием двух итерационных методов. В разных областях изменения параметра $\lambda$ используется тот или иной метод или же их комбинашия. Первый метод применялся авторами в работе [13]. Идея 
этого метода основывается на том, что для локализованного решения уравнения (7) значение $u(0)$ зависит от параметра $\lambda$ и поэтому, если фиксировать значение $u(0)$, задача сводится к отысканию параметра $\lambda$. На первом этапе нелинейная система уравнений заменяется линейной однородной системой. Для уравнений с квадратичной нелинейностью эта замена имеет вид $u_{i}^{2} \rightarrow \tilde{u}_{i} u_{i}$, где $\tilde{u}_{i}$ - значения искомых величин $u_{i}$ на предыдущей итерации. Далее решается задача на отыскание собственного значения $\lambda$ и собственного вектора $\left\{u_{i}\right\}$, который нормируется условием $u_{0}=u(0)$. После этого производится замена $u_{i} \rightarrow \tilde{u}_{i}$ и выполняется следуюшая итерация. Во втором методе параметр $\lambda$ фиксирован и для решения системы нелинейных уравнений применяется классическая итерационная процедура в сочетании с линеаризацией Ньютона [14].

Как упоминалось выше, для численного решения уравнения (7) используется асимптотика $u(x) \sim c / x^{2}, x \rightarrow \pm \infty$. Покажем справедливость этой асимптотики и найдем зависимость коэффициента $c$ от параметра $\lambda$. Выделим явно линейную часть функции $g(u)$ в исходном уравнении (6), представив ее в виде $g(u)=-u+f(u)$, поскольку именно линейная часть определяет поведение решения при $x \rightarrow \pm \infty$. Применим преобразование Фурье к нелинейному уравнению (7). Полагая

$$
U(k)=\frac{1}{2 \pi} \int_{-\infty}^{\infty} u(x) e^{-i k x} d x
$$

после несложных преобразований получаем

$$
U(k)=\frac{F(k)}{k^{2}+1+\lambda|k|},
$$

где

$$
F(k)=\frac{1}{2 \pi} \int_{-\infty}^{\infty} f(u) e^{-i k x} d x .
$$

Если симметрия уравнения допускает четные решения уравнения (6) $u(-x)=u(x)$, то для фурье-образа $F(k)$ таких решений выполняются соотношения

$$
F^{*}(k)=F(-k), \quad F(-k)=F(k) .
$$

Если же нелинейная функция $f(u)$ допускает сушествование нечетных решений уравнения (6) $u(-x)=-u(x)$, то для них имеют место следуюшие соотношения:

$$
F^{*}(k)=F(-k), \quad F(-k)=-F(k) .
$$

Совершая обратное преобразование Фурье для четных решений с учетом (13) получаем

$$
u(x)=2 \int_{0}^{\infty} \frac{F(k) \cos k x}{k^{2}+1+\lambda k} d k,
$$

а для нечетных решений уравнения (6) с учетом (14) имеем

$$
u(x)=2 i \int_{0}^{\infty} \frac{F(k) \sin k x}{k^{2}+1+\lambda k} d k .
$$


Для нахож дения поведения интегралов (15), (16) при $x \rightarrow \pm \infty$ используем метод стационарной фазы Кельвина [15], называемый также методом критических точек. Разлагаем вспомогательный интеграл $J(x)$ в ряд по малой величине $1 / x$ с помощью интегрирования по частям:

$$
J(x)=\int_{0}^{\infty} V(k) e^{i k x} d k=\sum_{n=0}^{\infty}\left(\frac{i}{x}\right)^{n+1} V^{(n)}(+0),
$$

где $V^{(n)}(+0)-n$-я производная от функции $V(k)$. Выделяя из интеграла $(17)$ действительную и мнимую части, получаем разложение для четного решения (15) в виде

$$
u(x)=-\frac{2}{x^{2}}\left(F^{\prime}(+0)-\lambda F(+0)\right)+O\left(x^{-4}\right)
$$

и для нечетного решения (16) в виде

$$
u(x)=\frac{2 i}{x} F(+0)-\frac{2 i}{x^{3}}\left(F^{\prime \prime}(+0)+2 \lambda F^{\prime}(+0)-2\left(1+\lambda^{2}\right) F(+0)\right)+O\left(x^{-5}\right) .
$$

Если нелинейная функция $f(u)$ может быть разложена в степенной ряд $f=a_{2} u^{2}+$ $a_{3} u^{3}+\ldots$, то, используя свойства свертки для фурье-образа произведения двух функций, мы легко покажем, что функция $F(k)$ и ее младшие производные $F^{\prime}(k), F^{\prime \prime}(k)$ непрерывны в точке $k=0$, хотя фурье-образ $U(k)$ функции $u(x)$ в точке $k=0$ может иметь разрывы или изломы, характерные для функций $u(x)$ со степенной асимптотикой. Учитьвая теперь свойства симметрии (13), (14), получаем окончательно асимптотическое разложение четного решения

$$
u(x)=2 \lambda F(0) \frac{1}{x^{2}}+O\left(x^{-4}\right)
$$

и нечетного решения

$$
u(x)=-4 i \lambda F^{\prime}(0) \frac{1}{x^{3}}+O\left(x^{-5}\right) .
$$

Таким образом, асимптотика $u(x) \sim c / x^{2}, x \rightarrow \pm \infty$, используемая для получения четных решений уравнения (7), находит свое подтверждение в приведенном анализе. Более того, т.к. для функции $f(u)=u^{2}$ фурье-образ $F(0)>0$, то знак коэффициента $c$ в асимптотике определяется знаком параметра $\lambda$. Асимптотика (20) согласуется с аналитическим решением (9), а также подтверждается в процессе численного расчета.

Численное решение уравнения (7) с асимптотикой (20) при произвольных положительных значениях параметра $\lambda$ показало, что решение $u(x)$ имеет колоколообразньй вид, как показано это на рис. 1 . С увеличением $\lambda$ значение амплитуды $u(0)$ возрастает и стремится к пределу $u(0)=2$, как это видно из формулы $(11)$, а ширина колокола стремится к бесконечности.

В случае отрицательных значений $\lambda$ характер поведения локализованных решений уравнения (7) меняется. Возникает колебательная структура, причем число колебаний функции $u(x)$ растет с уменьшением $\lambda$, и при $\lambda \rightarrow-2$ число колебаний стремится к бесконечности. По сути дела, в этом пределе решение преврашается в косинусоиду $u(x)=$ $A \cos x$ с амплитудой $A$, стремящейся к нулю, как это видно из рис. 2 . На такое поведение решения при $\lambda \rightarrow-2$ указывает также структура фурье-преобразования (12). Как 


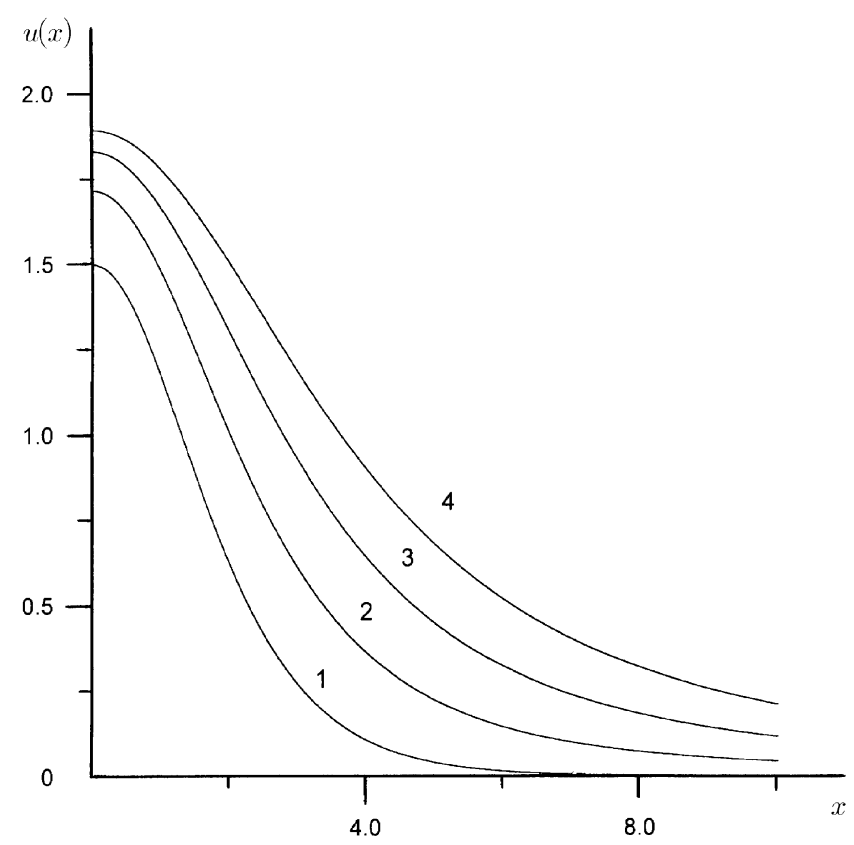

Рис. 1. Четные решения уравнения (7) для положительных значений координаты $x$ при $\lambda>0$ : кривая $1-\lambda=0$, кривая $2-\lambda=1$, кривая $3-\lambda=2$, кривая $4-\lambda=3$.

видно из этой формулы, при $-2<\lambda<0$ фурье-образ $U(k)$ искомого решения $u(x)$ имеет простые полюсы в точках комплексной плоскости

$$
k_{1,2}^{+}=\frac{1}{2}(\lambda \pm i \Delta), \quad k_{1,2}^{-}=\frac{1}{2}(-\lambda \pm i \Delta), \quad \Delta=\sqrt{4-\lambda^{2}} .
$$

Вклад в решение $u(x)$ от этих полюсов определяет колебательный предасимптотический член согласно формуле

$$
u(x)=A(\lambda) \cos \left(\frac{\lambda x}{2}+\alpha(\lambda)\right) e^{-\frac{\Delta x}{2}}+u_{p}(x),
$$

в которой коэффициенты $A(\lambda)$ и $\alpha(\lambda)$ стремятся к нулю при $\lambda \rightarrow-2$. В этой формуле через $u_{p}(x)$ обозначена часть решения, имеюшая правильную степенную асимптотику $u_{p}(x) \sim c / x^{2}$. Численный расчет показывает, что в рассматриваемом пределе $\lambda \rightarrow-2$ член $u_{p}(x)$ в выражении (23) при заданном $x$ стремится к нулю быстрее, чем колебательный член.

Проведенное выше рассмотрение относилось к области изменения параметра $-2<$ $\lambda<\infty$. Если $\lambda \leq-2$, то фурье-образ $U(k)$ имеет полюсы на действительной оси $k$. Любой способ обхода этих полюсов приводит к появлению в решении $u(x)$ незатухаюших колебательных членов. Таким образом, предположение о существовании локализованных решений уравнения (7) для значений параметра $\lambda \leq-2$ приводит к противоречию. Поиск локализованных решений в интервале $\lambda \leq-2$ численным методом также не увенчался успехом. Для систематизации четных локализованных решений уравнения (7) 


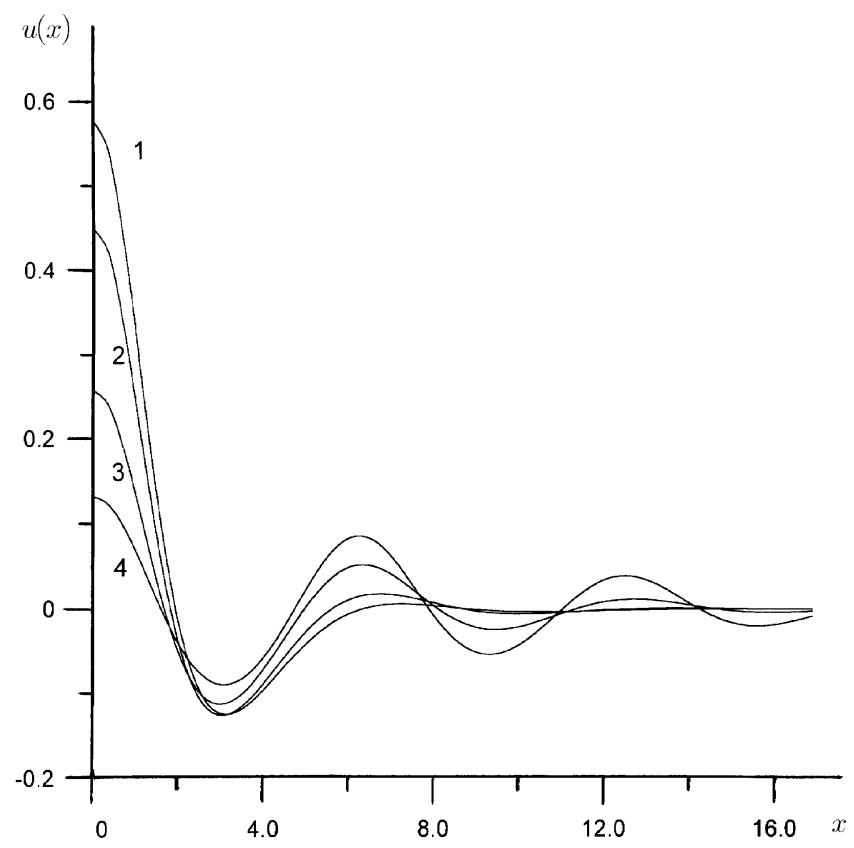

Рис. 2. Четные решения уравнения (7) для положительных значений координаты $x$ при $\lambda<0$ : кривая $1-\lambda=-1.68$, кривая $2-\lambda=-1.8$, кривая $3-\lambda=-1.94$, кривая $4-$ $\lambda=-1.985$.

удобно в области сушествования таких решений изобразить зависимости их начальной амплитуды $u(0)$ от параметра $\lambda$, как показано на рис. 3 , там же представлены еще две ветви локализованных решений уравнения $(7)$, для которых амплитуда $u(0)<0$. Эти ветви существуют в интервале значений параметра $-2<\lambda \leq-1.5$, а в точке $\lambda=-1.5$ они сливаются. На рис. 4 показаны решения уравнения (7), относяшиеся к ветвям II и III для $\lambda=-1.75$.

\section{3. НЕЛОКАЛЬНОЕ УРАВНЕНИЕ СИНУС-ГОР ДОН}

Рассмотрим уравнение $(6)$ с нелинейной частью $g(u)=\sin u$, т.е.

$$
u_{x x}+\sin u=\lambda K[u], \quad K[u]=\frac{1}{\pi} \int_{-\infty}^{\infty} \frac{u^{\prime}\left(x^{\prime}\right)}{x-x^{\prime}} d x^{\prime},
$$

где $u(x)$ - нечетная функция с граничными условиями типа волны переброса ("кинк"), когда $u(x) \rightarrow \pm \pi$ при $x \rightarrow \pm \infty$. Для нахождения асимптотики искомого решения применим преобразование Фурье. Однако интеграл Фурье для функции $u(x)$ расходится из-за выбранных граничных условий. Чтобы обойти эту трудность, введем вспомогательную функцию $w(x)$ с нулевыми граничными условиями:

$$
w(x)=u(x)-2 \operatorname{arctg} \frac{x}{a},
$$




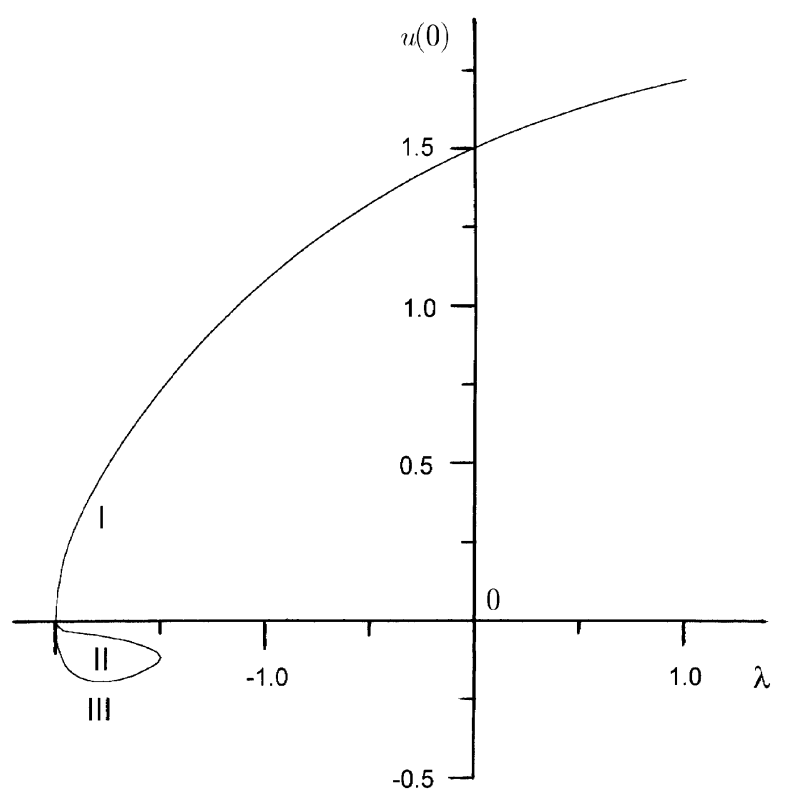

Рис. 3. Зависимость амплитуды $u(0)$ решения уравнения (7) от параметра $\lambda$ для трех его ветвей I, II, III.

где $a$ - произвольный параметр. Нелинейная часть уравнения (24) в этом случае принимает вид

$$
\sin u=\frac{2 x a}{a^{2}+x^{2}} \cos w+\frac{a^{2}-x^{2}}{a^{2}+x^{2}} \sin w .
$$

С ледуя методу, изложенному в предыдушем разделе, выделим из нелинейного члена (26) линейную часть $w$ следующим образом:

$$
\sin u=-w+\frac{2 x a}{a^{2}+x^{2}}+f(w),
$$

где $f(w)$ содержит члены, убывающие при $x \rightarrow \pm \infty$ быстрее линейного члена $w$, а именно

$$
f(w)=\frac{2 a^{2}}{a^{2}+x^{2}} w-\frac{x a}{a^{2}+x^{2}} w^{2}+\cdots
$$

Далее переписываем уравнение (24) для новой функции $w$ и совершаем преобразование Фурье. В результате получаем

$$
W(k)=\frac{F(k)+i(k-a \operatorname{sign}(k)+\lambda \operatorname{sign}(k)) e^{-|a k|}}{k^{2}+1+\lambda|k|},
$$

где $W(k), F(k)$ - фурье-образы функций $w(x), f(w)$. Применяя метод стационарной фазы $(17)$ и учитывая, что $F(+0)=0$, получаем асимптотику для функции $w$

$$
w(x)=\frac{2 i}{x} W(+0)+O\left(x^{-3}\right)=\frac{2}{x}(a-\lambda)+O\left(x^{-3}\right) .
$$




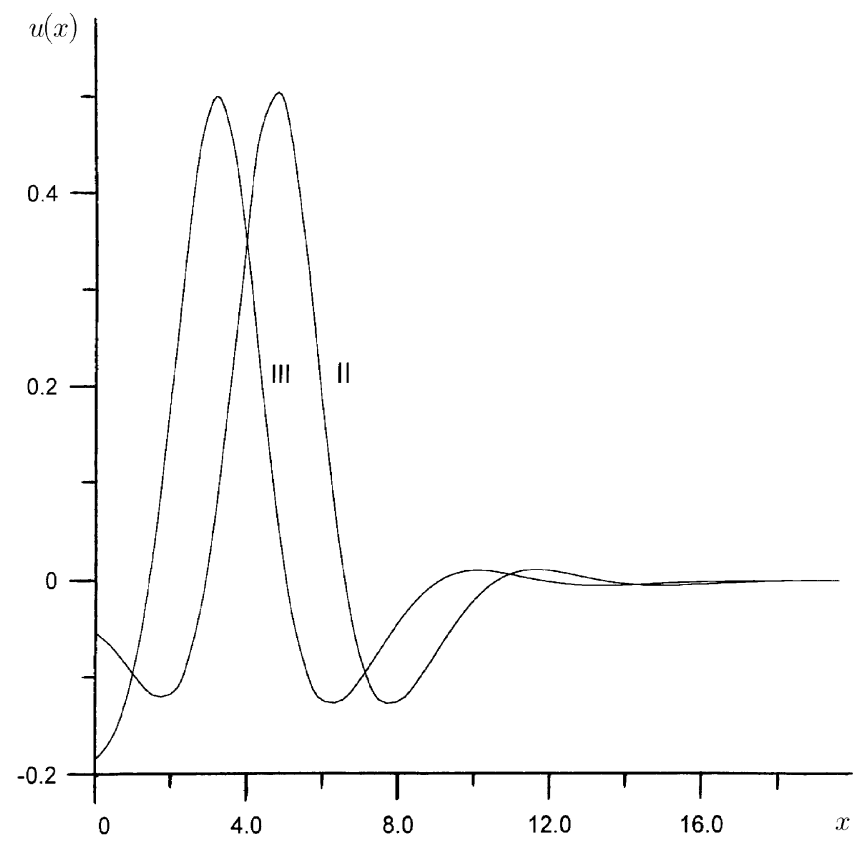

Рис. 4. Решения уравнения (7) для ветвей II и III при значении параметра $\lambda=-1.75$.

Используя асимптотическое разложение функции $\operatorname{arctg}(x / a)=\pi / 2-a / x+O\left(1 / x^{3}\right)$ и учитывая связь $(25)$, находим искомую асимптотику для функции $u(x)$ при $x \rightarrow+\infty$ :

$$
u(x)=\pi-\frac{2 \lambda}{x}+O\left(x^{-3}\right)
$$

Заметим, что произвольный параметр $а$ выпадает из окончательного выражения. Вмес$\operatorname{To} \operatorname{arctg}(x / a)$ можно было выбрать любую другую функцию с такими же граничными условиями.

Если параметр $\lambda$ равен 0, то уравнение (24) не содержит нелокального члена и преврашается в уравнение синус-Гордон, которое имеет хорошо известное солитонное решение

$$
u(x)=4 \operatorname{arctg}\left(e^{x}\right)-\pi .
$$

При больших значениях параметра $\lambda$ решение уравнения (24) можно представить в виде разложения по малому параметру $1 / \lambda$ :

$$
u(x)=u_{0}(x / \lambda)+\frac{1}{\lambda} u_{1}(1 / \lambda)+\cdots,
$$

где $u_{0}(\xi)$ удовлетворяет интегральному уравнению

$$
\sin u_{0}(\xi)=\frac{1}{\pi} \int_{-\infty}^{\infty} \frac{u_{0}(\xi)-u_{0}\left(\xi^{\prime}\right)}{\left(\xi-\xi^{\prime}\right)^{2}} d \xi^{\prime}
$$




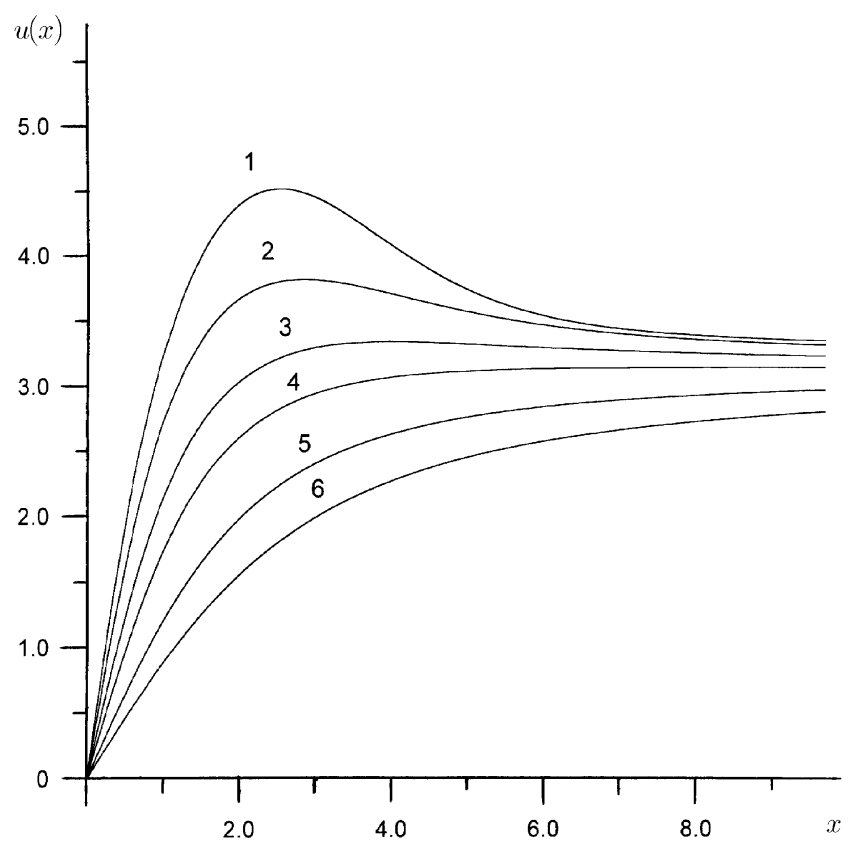

Рис. 5. Первая ветвь нечетного решения уравнения (24) при $x>0$ для различных значений параметра $\lambda$ : кривая $1-\lambda=-1$, кривая $2-\lambda=-0.8$, кривая $3-\lambda=-0.4$, кривая $4-\lambda=0$, кривая $5-\lambda=0.8$, кривая $6-\lambda=1.6$.

в котором введена переменная $\xi=x / \lambda$. Это уравнение имеет известное решение с нужными граничными условиями

$$
u_{0}(\xi)=2 \operatorname{arctg} \xi
$$

Таким образом, в первом приближении для $\lambda \gg 1$ имеем решение

$$
u(x)=2 \operatorname{arctg} \frac{x}{\lambda} .
$$

Для произвольных значений параметра $\lambda$ решение уравнения (24) с асимптотикой (28) находилось численно с использованием методов, изложенных в предыдущем разделе. При изменении $\lambda$ от 0 до $\infty$ решение уравнения (24) деформируется непрерывным образом от чистого кинка (29) к решению (31). Для отрицательных значений параметра $\lambda<0$ в соответствии с асимптотикой $(28)$ решение имеет всплеск над уровнем $u(x)=\pi$. На рис. 5 показана эволюция решения $u(x)$ с изменением параметра $\lambda$ в интервале $-1 \leq$ $\lambda<\infty$. При уменьшении $\lambda$ от нуля до значения -1 всплеск растет, так что максимальное значение $u_{\max }$ приближается к уровню $u(x)=3 \pi / 2$. При дальнейшем уменьшении параметра $\lambda$ происходит срыв с этого типа решения, и мы попадаем на другую ветвь решений уравнения (24). На рис. 6 показаны решения для еще одной такой ветви. Имеются и другие ветви нечетных локализованных решений с большим числом колебаний в области локализации. Аналогично уравнению (7) здесь тоже не удалось найти локализованных решений уравнения (24) для значений параметра $\lambda \leq-2$, поэтому можно 


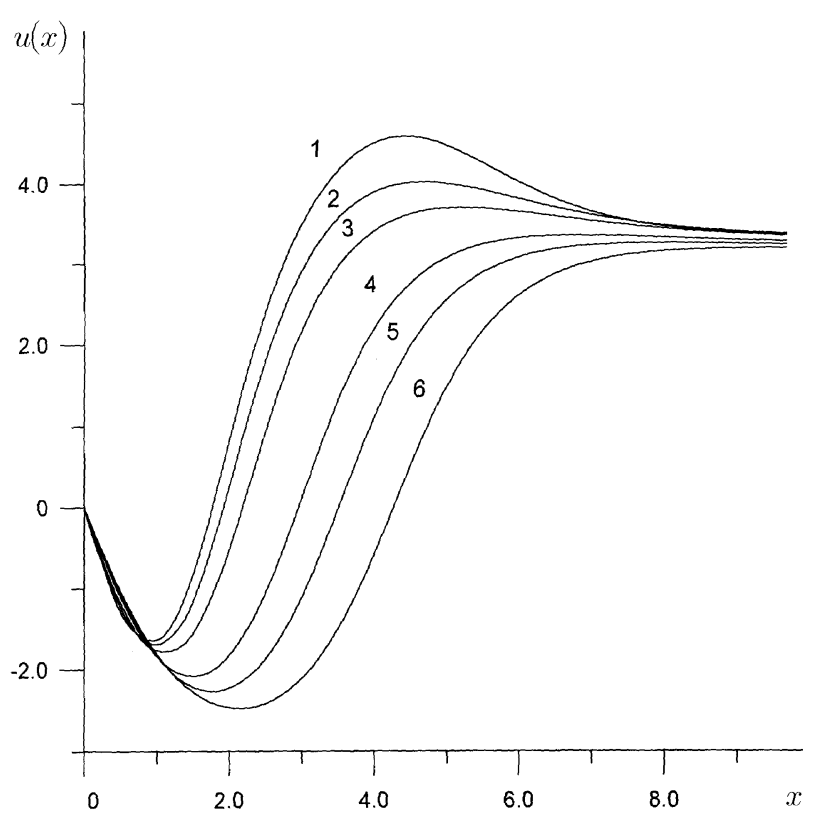

Рис. 6. Вторая ветвь нечетного решения уравнения (24) при $x>0$ для различных значений параметра $\lambda$ : кривая $1-\lambda=-1.16$, кривая $2-\lambda=-1.04$, кривая $3-\lambda=-0.86$, кривая $4-\lambda=-0.5$, кривая $5-\lambda=-0.34$, кривая $6-\lambda=-0.2$.

высказать предположение об отсутствии локализованных решений нелокальных уравнений $(7),(24)$ с заданным интегральным ядром в этой области изменения параметра $\lambda$.

\section{4. ЗАКЛЮЧЕНИЕ}

Таким образом, анализ нелинейных уравнений типа уравнения нелинейного маятника в вязкой среде с нелокальным взаимодействием показал, что его локализованные решения различаются как асимптотикой вблизи положений равновесия на бесконечности, так и характером немонотонности в области локализации в зависимости от симметрии решения, знака и величины параметра $\lambda$, характеризуюшего нелокальное взаимодействие. Четные решения уравнения с квадратичной нелинейностью имеют три ветви солитонов. Амплитуда солитонов первого типа монотонно уменьшается до нуля от центра к периферии в области положительных значений параметра $\lambda$. Асимптотика этого решения имеет вид $u \sim 1 / \tau^{2}$, что согласуется с известными результатами работы [6]. При отрицательных значениях $\lambda$ появляются немонотонные осцилляции в области локализации, а амплитуда в центре солитона падает до нуля при приближении к критической величине параметра нелокальности $\lambda=-2$. В области $-2<\lambda \leq-1.5$ найдены еше две ветви четных локализованных решений, различаюшихся знаком кривизны в центре солитона и имеюших отрицательные амплитуды. Устойчивость этих решений нами не рассматривалась. 
Нечетные решения уравнения типа синус-Гордон с интегральным ядром $G=$ $\lambda /\left(\tau-\tau^{\prime}\right)$ имеют асимптотику $\left|u-u_{ \pm \infty}\right| \sim 1 / \tau$ вблизи равновесия на бесконечности. Здесь также имеется несколько различающихся типов солитонных решений в зависимости от знака и величины параметра нелокальности $\lambda$. В положительной области изменения этого параметра сушествуют монотонные локализованные решения типа волны переброса. При $\lambda<0$ в этих решениях возникает немонотонность в виде перегиба на крыльях солитона. Последний тип солитонов близок к решениям, описываюшим динамику блоховских линий в доменной границе [10], а также магнитных солитонов одноосного ферромагнетика вблизи поля насыщения в перпендикулярном направлении [11].

Предложенный метод анализа нелинейных уравнений с нелокальным ядром типа $G=$ $\lambda /\left(\tau-\tau^{\prime}\right)$ может быть легко обобщен на случай ядра $G=\lambda /\left(\tau-\tau^{\prime}\right)^{n}$, где $n=2,3,4$, и на другие ядра более общего вида, которые могут применяться для описания динамики солитонов в диссипативной среде.

Авторы благодарят проф. В. М. Елеонского за полезные обсуждения.

Работа поддержана Международным научным фондом (ISF) (грант J8P100) и фондом РФФИ (грант 95-02-03737-а, грант 97-01-00528).

\section{Список литературы}

[1] Yu. M. Aliev, V.P. Silin, S. A. Urupin. Superconductivity. 1992. V. 5. P. 230.

[2] A. Gurevich. Phys. Rev. B. 1992. V. 46. P. 230.

[3] G. L. Alfimov, A.F. Popkov. Phys. Rev. B. 1995. V. 52. P. 4503.

[4] Yu. M. Ivanchenko, T. K. Soboleva. Phys. Lett. A. 1990. V. 147. P. 65.

[5] R. G. Mints, I. B. Shapiro. Phys. Rev. B. 1995. V. 51. P. 3054.

[6] A. O. Caldeira, A. J. Leggett. Ann. Phys. (NY). 1984. V. 149. P. 374.

[7] A. O. Caldeira, A. J. Leggett. Physica. A. 1983. V. 121. P. 587.

[8] A. Garg. Phys. Rev. Lett. 1993. V. 70. № 10. P. 1541.

[9] A. Garg, G. H. Kim. Phys. Rev. B. 1991. V. 43. P. 712

[10] C. E. Stamp. Physica. B. 1994. V. 197. P. 133.

[11] A. P. Malozemoff, J. C. Slonszewskii. Magnetic Domain Walls in Bubble Materials. New York: Acad. Press, 1979.

[12] Н. Е. Кулагин, А.Ф. Попков. Письма в ЖЭТФ. 1986. Т. 43. № 4. С. 197.

[13] B. E. Ivanov, N. E. Kulagin, K. A. Safaruan. Physica. B. 1994. V. 202. P. 193.

[14] Н. Н. Ахмедиев, В. И. Корнеев, Ю. В. Кузьменко. ЖЭТФ. 1985. Т. 88. № 1. С. 107.

[15] Ф. Олвер. Асимптотика и специальные функции. М.: Наука, 1990.

Поступила в редакцию $10 . \mathrm{IX} .1997$ г. 